\title{
Pediatric skull fracture diagnosis: should 3D CT reconstructions be added as routine imaging?
}

\author{
Gunes Orman, MD, ${ }^{1}$ Matthias W. Wagner, MD, ${ }^{1}$ Daniel Seeburg, MD, ${ }^{1}$ Carlos A. Zamora, MD, PhD, ${ }^{2}$ \\ Alexander Oshmyansky, MD, PhD, ${ }^{1}$ Aylin Tekes, MD, ${ }^{1}$ Andrea Poretti, MD, ${ }^{1}$ George I. Jallo, MD, ${ }^{3}$ \\ Thierry A. G. M. Huisman, MD, ${ }^{1}$ and Thangamadhan Bosemani, MD'1
}

\begin{abstract}
${ }^{1}$ Section of Pediatric Neuroradiology, Division of Pediatric Radiology, and 2Division of Neuroradiology, Russell H. Morgan Department of Radiology and Radiological Science; and ${ }^{3}$ Division of Pediatric Neurosurgery, The Johns Hopkins University School of Medicine, Baltimore, Maryland
\end{abstract}

\begin{abstract}
OBJECT The authors compared the efficacy of combining 2D+3D CT reconstructions with standard 2D CT images in the diagnosis of linear skull fractures in children with head trauma.

METHODS This was a retrospective evaluation of consecutive head CT studies of children presenting with head trauma. Two experienced pediatric neuroradiologists in consensus created the standard of reference. Three readers independently evaluated the 2D CT images alone and then in combination with the 3D reconstructions for the diagnosis of linear skull fractures. Sensitivity and specificity in the diagnosis of linear skull fractures utilizing 2D and 2D+3D CT in combination were measured for children less than 2 years of age and for all children for analysis by the 3 readers.
\end{abstract}

RESULTS Included in the study were 250 consecutive CT studies of 250 patients ( 167 boys and 83 girls). The mean age of the children was 7.82 years (range 4 days to 17.4 years). 2D+3D CT combined had a higher sensitivity and specificity $(83.9 \%$ and $97.1 \%$, respectively) compared with $2 \mathrm{D}$ alone $(78.2 \%$ and $92.8 \%$, respectively) with statistical significance for specificity $(p<0.05)$ in children less than 2 years of age. 2D+3D CT combined had a higher sensitivity and specificity $(81.3 \%$ and $90.5 \%$, respectively) compared with $2 \mathrm{D}$ alone $(74.5 \%$ and $89.1 \%$, respectively) with statistical significance for sensitivity $(p<0.05)$ in all children.

CONCLUSIONS In this study, 2D+3D CT in combination showed increased sensitivity in the diagnosis of linear skull fractures in all children and increased specificity in children less than 2 years of age. In children less than 2 years of age, added confidence in the interpretation of fractures by distinguishing them from sutures may have a significant implication in the setting of nonaccidental trauma. Furthermore, 3D CT is available at no added cost, scan time, or radiation exposure, providing trainees and clinicians with limited experience an additional valuable tool for routine imaging of pediatric head trauma.

http://thejns.org/doi/abs/10.3171/2015.3.PEDS1553

KEY WORDS computed tomography; head trauma; children; skull fracture

$\mathrm{S}$ KULL fractures occur in up to $2 \%$ of all children ${ }^{2}$ and $11 \%$ of children under 2 years of age following head trauma. ${ }^{5}$ Head CT identifies posttraumatic skull fractures with high sensitivity. ${ }^{15}$ Routine 2D CT images may not be sufficient to identify subtle fractures or linear fractures orienting in the axial plane on images or reconstructions. ${ }^{17}$

Skull fractures may be linear, depressed, diastatic, or basilar (skull base). Linear fractures account for ap- proximately $75 \%$ of all fractures..$^{10,15}$ The parietal bone is most commonly fractured (approximately $60 \%-70 \%$ of the time)..$^{10,15}$ Linear skull fractures are associated with intracranial injury in $15 \%-30 \%$ of patients. Conversely, $40 \%-100 \%$ of intracranial injuries have an associated skull fracture. ${ }^{15}$ Intracranial injury may be primary or secondary.$^{10}$ Primary injury as a consequence of direct impact includes, for example, epidural and subdural hemorrhage, diffuse axonal injury, and cortical contusion. ${ }^{10}$ Secondary

ABBREVIATIONS MDCT = multidetector CT; MIP = maximum intensity projection; $\mathrm{VR}=$ volume rendered. SUBMITTED January 25, 2015. ACCEPTED March 20, 2015. INCLUDE WHEN CITING Published online July 17, 2015; DOI: 10.3171/2015.3.PEDS1553.

DISCLOSURE The authors report no conflict of interest concerning the materials or methods used in this study or the findings specified in this paper. 
injury is a complication of the primary injury (e.g., stroke due to hematoma-related herniation) and may occur with a delay in time after the initial trauma. Intracranial injury is a leading cause of mortality and morbidity in children. ${ }^{6}$ A skull fracture may be an indicator of intracranial injury; hence, an accurate diagnosis in pediatric head trauma is important. ${ }^{9,16}$

Routine imaging evaluation in pediatric head trauma includes assessment of the following: 1) CT scout image, 2) axial $2 \mathrm{D}$ images in bone and soft-tissue windows, and 3) 2D multiplanar reformatted images in both coronal and sagittal planes. The presence of scalp swelling or hematoma and intracranial injury such as contusion or hemorrhage may also be helpful in identifying a subtle fracture.

Multidetector CT (MDCT) provides an isotropic volume data set from which 2D, multiplanar, and 3D reconstructions can be obtained. ${ }^{11}$ The high sensitivity of highresolution volume-rendered (VR) 3D CT in detecting skull fractures was reported in 6 pediatric human cadaver skulls after they were exposed to head drop tests. ${ }^{8}$ The 3D data set can be made available by simple postprocessing techniques immediately after the $2 \mathrm{D}$ image acquisition and is a potential valuable source of information with no added cost, scan time, or radiation exposure. The postprocessing time for preparing the 3D data set by the CT technologist is approximately 5 minutes and can be performed in a workstation remote from the scanner, hence not impacting the workflow.

2D CT imaging in bone kernels with multiplanar reformats is the current standard of practice. The potential added value of 3D CT has not been assessed. The goal of this study was to compare sensitivity and specificity between combined 3D+2D CT image data sets and 2D CT alone in detecting linear skull fractures in a large cohort of children with head trauma. The image evaluation was performed by junior radiologists (resident, fellow, and junior faculty member) and measured against the standard of reference created by 2 experienced pediatric neuroradiologists.

\section{Methods}

The Johns Hopkins University School of Medicine institutional research ethics board approved this study.

\section{Study Population}

The study inclusion criteria were: 1) history of minor or major head trauma, 2) head CT studies performed at our tertiary children's hospital, and 3) age younger than 18 years at scanning. The exclusion criteria were: 1) outside CT studies submitted for second opinion interpretation, and 2) fractures other than linear fractures. Head CT studies were collected through an electronic search of our pediatric neuroradiology database covering the time period between February 1, 2011, and April 30, 2013. The first 250 consecutive patients matching the inclusion criteria were selected for image analysis.

\section{CT Protocol}

The examinations were obtained on a commercially available $128 \times 2$-detector system (Somatom Definition
Flash, Siemens) or 64-detector system (Somatom Sensation, Siemens) using our institutional pediatric CT protocol, without intravenous injection of a contrast agent for acute head trauma, with the parameters summarized in Table 1.

Image reconstruction was performed by the CT technologist using an FDA-approved medical workstation (Leonardo, Syngo MMWP, Siemens). All examinations were subjected to VR 3D reconstruction algorithms with $360^{\circ}$ feet-to-brain spin and $360^{\circ}$ left-to-right spin for standardization processes and then stored on the PACS system.

\section{Image Analysis}

The standard of reference for diagnosis of a fracture was established by 2 experienced pediatric neuroradiologists in consensus (T.A.G.M.H. with 20 years and A.T. with 8 years of experience in pediatric neuroradiology). Neither of them participated in the study as study readers. The 2D and 3D CT image data sets were reviewed in bone windows to establish the standard of reference. The available radiological interpretations in the electronic patient records were not taken into consideration for establishing the standard of reference.

The 3 readers made independent evaluations. All CT examinations were independently evaluated by a thirdyear resident (D.S., Reader 3), a neuroradiology fellow (C.A.Z., Reader 2), and a pediatric neuroradiology attending with 1 year of experience (T.B., Reader 1). These 3 readers independently evaluated the $2 \mathrm{D}$ CT images initially and subsequently both the $2 \mathrm{D}+3 \mathrm{D}$ CT images in combination to yield 2 separate readings each. There was a 4 -week time lapse between the 2 readings. For each evaluation, the readers determined: 1) the presence or absence of a linear skull fracture, and 2) if a fracture was present, the description of the involved bones (frontal, parietal, temporal, and/or occipital). The readers were also given the following options to show their certainty for positive findings: 1) definite fracture, and 2) possible fracture. To eliminate reader fatigue, the evaluation was limited to a maximum of $50 \mathrm{CT}$ studies per session. The first author (G.O.) assisted each of the 3 readers during these sessions by opening only the relevant images ( $2 \mathrm{D}$ during the initial session, and subsequently both $2 \mathrm{D}+3 \mathrm{D}$ CT image data sets) in bone kernels during each session and entered the data in an anonymized worksheet.

TABLE 1. Axial head CT protocol for pediatric trauma patients

\begin{tabular}{ll}
\hline \multicolumn{1}{c}{ Element } & \multicolumn{1}{c}{ Parameters } \\
\hline Tube parameters & $\begin{array}{c}\text { Tube voltage } 120 \mathrm{kV} \text {, tube current } 380 \text { reference } \\
\mathrm{mA} \text {, rotation time } 1.0 \mathrm{sec}\end{array}$ \\
$\begin{array}{ll}\text { Dose modulation } & \text { CARE Child software package (Siemens), dose } \\
\text { enabled }\end{array}$ \\
\hline Collimation & $2 \times 128$ dual source, 64 \\
\hline Reconstructions & $\begin{array}{c}\text { Transverse orientation, } 0.75-\mathrm{mm} \text { section thickness, } \\
0.5 \text {-mm reconstruction interval, reconstruction } \\
\text { kernel H70 (bone) }\end{array}$ \\
\hline Field of view & $160 \times 160-250 \times 250 \mathrm{~mm}$ \\
\hline
\end{tabular}

CARE $=$ combined applications to reduce exposure. 


\section{Statistical Analysis}

The 2 independent evaluations $(2 \mathrm{D}$, and 2D+3D combined) from the 3 readers were analyzed individually and in combination. The decision to classify one fracture as a missed fracture (a fracture that was present according to the standard of reference, but not reported) was considered a false-negative result, and an overdiagnosed fracture (a fracture that was not present according to the standard of reference, but was reported) was considered a falsepositive result. The decision of a possible fracture interpretation was considered as a fracture for the purpose of statistical analysis. To measure sensitivity and specificity, specific decisions (true positive, false positive, true negative, or false negative) were correlated with total decisions for all 3 readers. In addition, for all 3 readers, sensitivity and specificity were also separately analyzed for children less than 2 years of age (sutures not yet closed). A 2-tailed t-test was performed to determine the statistical significance $(p<0.05)$ of whether a higher proportion of falsenegative or false-positive studies were present between the $2 \mathrm{D}$ alone and 2D+3D data sets for all 3 readers. To compare sensitivity and specificity, $2 \times 2$ contingency tables were used to assess the data, and a McNemar test was performed to compare the categories.

The individual misinterpretation numbers for all readers in all children were analyzed. Using $2 \times 2$ contingency tables, the data were assessed between each pair of readers (Reader 1 and Reader 2, Reader 2 and Reader 3, and Reader 1 and Reader 3), and a McNemar test was used for comparison of different categories.

\section{Results}

The study included 250 consecutive examinations in 250 patients (167 boys and 83 girls). The mean age of the children was 7.82 years (range 4 days to 17.4 years).

According to the standard of reference, 82 skull fractures were diagnosed in 76 children. A total of 174 children had no fractures; 38 of the 82 fractures $(46.3 \%)$ were diagnosed in 32 of the 76 children (42.1\%) less than 2 years of age. The distribution of each fracture with regard to its location was as follows: 35 of 82 were parietal (42.7\%), 20 of 82 were frontal (24.4\%), 14 of 82 were occipital (17.1\%), 11 of 82 were temporal (13.4), 1 of 82 was parietal and temporal (1.2\%), and 1 of 82 was parietal and occipital $(1.2 \%)$.

Each reader had a total of 512 decisions to make: 164 fracture decisions ( 82 fractures on 2D CT, and 82 fractures on $2 \mathrm{D}+3 \mathrm{D} \mathrm{CT})$ and 348 no-fracture decisions (174 no-fracture decisions on 2D CT and 174 on 2D+3D CT). The total false-positive studies, total false-negative studies, and sensitivity and specificity of all 3 readers using $2 \mathrm{D}$ and 2D+3D CT for all children and for children less than 2 years of age are summarized in Table 2 , along with the statistical significances for the false-positive studies, falsenegative studies, sensitivity, and specificity of 2D+3D CT in comparison with 2D CT. In all children, sensitivity and false-negative studies $(\mathrm{p}<0.05)$ demonstrated statistical significance. In children less than 2 years of age, falsepositive studies, false-negative studies, and specificity $(\mathrm{p}<$ 0.05 ) demonstrated statistical significance.

The total (all 3 readers) rate of misinterpretation for 2D
TABLE 2. Sensitivity and specificity of all 3 readers combined*

\begin{tabular}{|c|c|c|c|c|c|c|}
\hline \multirow[b]{2}{*}{ Variable } & \multicolumn{3}{|c|}{ All Children } & \multicolumn{3}{|c|}{ Children <2 Yrs Old } \\
\hline & $2 \mathrm{D}$ & $2 D+3 D$ & $\begin{array}{c}p \\
\text { Value }\end{array}$ & $2 \mathrm{D}$ & $2 D+3 D$ & $\begin{array}{c}\mathrm{p} \\
\text { Value }\end{array}$ \\
\hline $\begin{array}{l}\text { False-negative } \\
\text { studies }\end{array}$ & $65 / 768$ & $45 / 768$ & 0.02 & $29 / 186$ & $21 / 186$ & 0.03 \\
\hline $\begin{array}{l}\text { False-positive } \\
\text { studies }\end{array}$ & $56 / 768$ & $50 / 768$ & 0.34 & $10 / 186$ & $4 / 186$ & 0.02 \\
\hline Sensitivity (\%) & 74.5 & 81.3 & 0.01 & 78.2 & 83.9 & 0.15 \\
\hline Specificity (\%) & 89.1 & 90.5 & 0.93 & 92.8 & 97.1 & 0.04 \\
\hline
\end{tabular}

* Boldface values are statistically significant $(p \leq 0.05)$.

CT alone (121 of 768 decisions [15.8\%]) was higher than that for 2D+3D CT (95 of 768 decisions [12.4\%]) in all children. The misinterpretations by the individual readers for all children are summarized in Table 3, along with the sensitivity and specificity rates of each individual reader for both 2D+3D and 2D alone. There were no statistically significant differences demonstrated between the individual readers for decisions made.

Missed fractures, or "undercalls" (false negatives), by all readers with regards to location are shown in Table 4. Parietal bone fractures (27 of 65 [41.5\%]) were the most frequently missed type on 2D CT. Overdiagnosed fractures, or "overcalls" (false positives), by all readers with regards to location are shown in Table 5. Temporal bone fractures were most frequently overcalled on 2D CT (23 of $56[41.1 \%])$ and 2D+3D CT (15 of 50 [30\%]).

\section{Discussion}

A variety of computer algorithms can generate $3 \mathrm{D}$ reconstructions of CT image data sets; the 3 most commonly used techniques are shaded surface display, maximum intensity projection (MIP), and VR. ${ }^{1}$ Previous studies have shown the utility and value of 3D head CT provided by different algorithms in the diagnosis of fractures in adults. ${ }^{4,12-14}$

In all children, we found that 2D+3D CT increased the sensitivity $(81.3 \%, \mathrm{p}<0.05)$ when compared with $2 \mathrm{D}$ CT only $(74.5 \%)$ in the diagnosis of linear skull fractures. In addition, fewer false-negative calls (or undercalls) with $2 \mathrm{D}+3 \mathrm{D}(\mathrm{n}=45)$ when compared with $2 \mathrm{D}$ alone $(\mathrm{n}=65)$ showed statistical significance $(p<0.05)$. The increased sensitivity and fewer false-negative calls of $2 \mathrm{D}+3 \mathrm{D}$ demonstrate its capability in detection of linear fractures. Linear fractures on 2D CT may be missed when they are

TABLE 3. Misinterpretations by individual readers in all children

\begin{tabular}{|c|c|c|c|c|c|c|c|c|}
\hline \multirow[b]{2}{*}{ Variable } & \multicolumn{4}{|c|}{$2 \mathrm{D}$} & \multicolumn{4}{|c|}{$2 D+3 D$} \\
\hline & $\mathrm{R} 1$ & $\mathrm{R} 2$ & R3 & Total & $\mathrm{R} 1$ & $\mathrm{R} 2$ & R3 & Total \\
\hline $\begin{array}{l}\text { False-negative } \\
\text { studies }\end{array}$ & 19 & 21 & 25 & 65 & 14 & 15 & 16 & 45 \\
\hline $\begin{array}{l}\text { False-positive } \\
\text { studies }\end{array}$ & 14 & 23 & 19 & 56 & 14 & 22 & 14 & 50 \\
\hline Sensitivity (\%) & 78.1 & 73.8 & 71.6 & & 82.9 & 80.0 & 80.9 & \\
\hline Specificity (\%) & 92.0 & 86.8 & 89.0 & & 92.0 & 87.3 & 92.0 & \\
\hline
\end{tabular}

R1 = Reader 1; R2 = Reader 2; R3 = Reader 3. 
TABLE 4. Percentage of missed fractures by all readers for all children with regards to fracture location

\begin{tabular}{lcccc}
\hline Technique & Frontal (\%) & Parietal (\%) & Temporal (\%) & Occipital (\%) \\
\hline 2D CT only & $12 / 65(18.5)$ & $27 / 65(41.5)$ & $12 / 65(18.5)$ & $14 / 65(21.5)$ \\
\hline 2D+3D CT & $10 / 45(22.2)$ & $13 / 45(28.9)$ & $13 / 45(28.9)$ & $9 / 45(20)$ \\
\hline
\end{tabular}

within the plane of the image reconstruction, and the addition of 3D should alleviate this problem (Figs. 1 and 2). The specificity of 2D+3D CT $(90.5 \%)$ in comparison with 2D only (89.1\%) did not have a statistically significant correlation, likely because complete sutural fusion in older children diminishes the uncertainty of sutures mimicking linear fractures and hence no difference in false-positive calls (overcalls).

In children less than 2 years of age, 2D+3D CT demonstrated increased sensitivity and specificity $(83.9 \%$ and $97.1 \%$, respectively) in detection of linear skull fractures in comparison with 2D CT alone (sensitivity $78.2 \%$ and specificity $92.8 \%)$. Statistical significance $(\mathrm{p}<0.05)$ was shown for specificity alone in children less than 2 years of age. In a previous study, 3D CT was found to be superior to plain radiography in the assessment of skull fractures in younger children with an incompletely ossified calvaria. ${ }^{7}$ In children less than 2 years of age, the presence of open sutures may increase the diagnostic uncertainty of the $2 \mathrm{D}$ CT data set. Figure 3 shows 3D reconstructions of the cranial sutures of 4 children at different ages. The addition of 3D to the 2D $\mathrm{CT}$ data set gives the reader increased confidence as sutures and other nonfracture-related linear lucencies such as vascular channels can be easily followed and distinguished from linear fractures, hence decreasing the overcalls (falsepositive rates; Figs. 4 and 5) and increasing specificity. In addition, for children less than 2 years of age, lower falsenegatives (undercalls) and lower false-positives (overcalls) were shown with $2 \mathrm{D}+3 \mathrm{D} \mathrm{CT}$ with statistical significance. The fewer false-negative results reflect the added confidence of the reader in using 2D+3D CT to identify subtle linear skull fractures in close relation to sutures in children less than 2 years of age. The accurate diagnosis of fracture in a child less than 2 years of age may be important in the setting of nonaccidental trauma.

The range of sensitivity $(71.6 \%-78.1 \%)$ for individual readers for $2 \mathrm{D}$ was lower when compared with the sensitivity (80\%-82.9\%) for 2D+3D CT. Reader 1, with the most experience, showed no significant difference in sensitivity between $2 \mathrm{D} \mathrm{CT}$ and $2 \mathrm{D}+3 \mathrm{D} \mathrm{CT}$. The addition of 3D is particularly helpful for trainees and radiologists with limited experience in the evaluation of pediatric CT studies. The benefit of 3D as a new postprocessing tool together with the increasing experience of the reader is demonstrated in this study.

Parietal bone fractures were most commonly missed

TABLE 5. Percentage of overdiagnosed fractures by all readers for all children with regards to location

\begin{tabular}{llllc}
\hline Technique & Frontal (\%) & Parietal (\%) & Temporal (\%) & Occipital (\%) \\
\hline 2D CT only & $12 / 56(21.4)$ & $14 / 56(25)$ & $23 / 56(41.1)$ & $7 / 56(12.5)$ \\
\hline 2D+3D CT & $11 / 50(22)$ & $14 / 50(28)$ & $15 / 50(30)$ & $10 / 50(20)$ \\
\hline
\end{tabular}
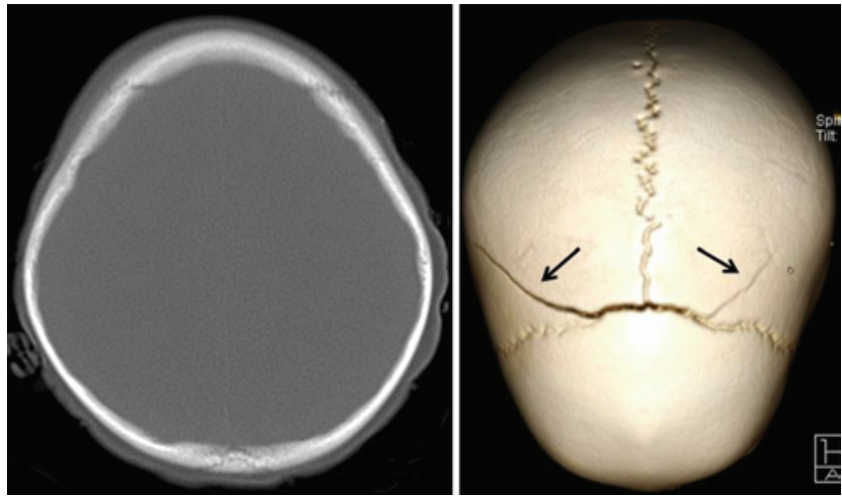

FIG. 1. Images obtained of a 23-month-old girl who presented after a motor vehicle accident in which she was a restrained back-seat passenger in a car seat, which was forward-facing. The 2D CT scan shows no fracture line (left), whereas the 3D CT scan (right) reveals bilateral nondisplaced fractures of parietal bones extending to the coronal suture (arrows). Figure is available in color online only.

on 2D CT alone (27 of 65 [41.5\%]), which is consistent with prior studies..$^{0,15}$ Temporal bone fractures were more commonly missed on 2D+3D CT (13 of 45 [28.9\%]) compared with 2D alone (12 of 65 [18.5\%]); 3D CT is not particularly helpful in the evaluation of temporal bone linear fractures. Temporal bone fractures were also most often overcalled on both 2D alone (23 of 56 [41.1\%]) and 2D+3D $\mathrm{CT}(15$ of $50[30 \%])$, which is likely related to the complex temporal bone anatomy and adjacent sutures.

Complex or depressed fractures may be more readily apparent clinically by a focal soft-tissue swelling or skullshape deformity. Linear skull fractures may not have significant scalp edema or swelling. The diagnosis of linear fractures is important, because it is an independent risk factor of intracranial injuries in children. ${ }^{3}$

The postprocessing time of $3 \mathrm{D} \mathrm{CT}$ is very short and does not add substantial indirect cost; however, its benefit of added information without additional radiation exposure in the setting of trauma has been shown in our study. Hence, it should be routinely used in the evaluation of pediatric head trauma. The efficacy of 3D CT for other clinical indications in pediatric head imaging has not been specifically evaluated.

The retrospective nature of the study and inclusion of only linear skull fractures are potential limitations. Each
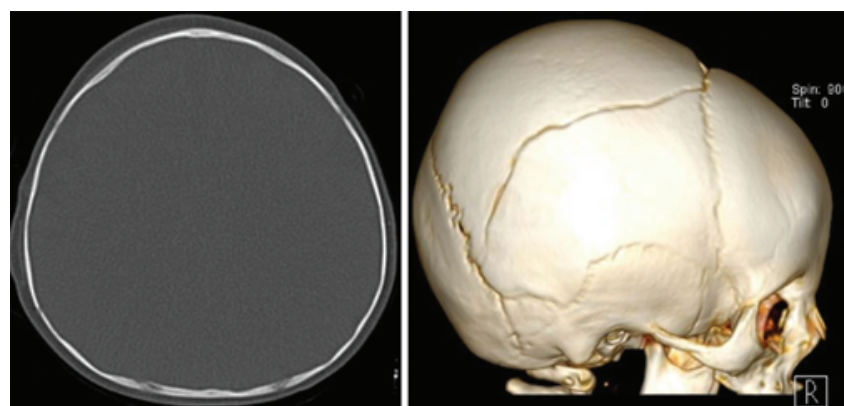

FIG. 2. Images of an 8-month-old boy who fell from a couch onto a hardwood floor. The 2D CT scan shows no evidence of a fracture (left), but the 3D CT scan (right) reveals a nondisplaced fracture through the right parietal bone extending posteriorly from the coronal suture. Figure is available in color online only. 

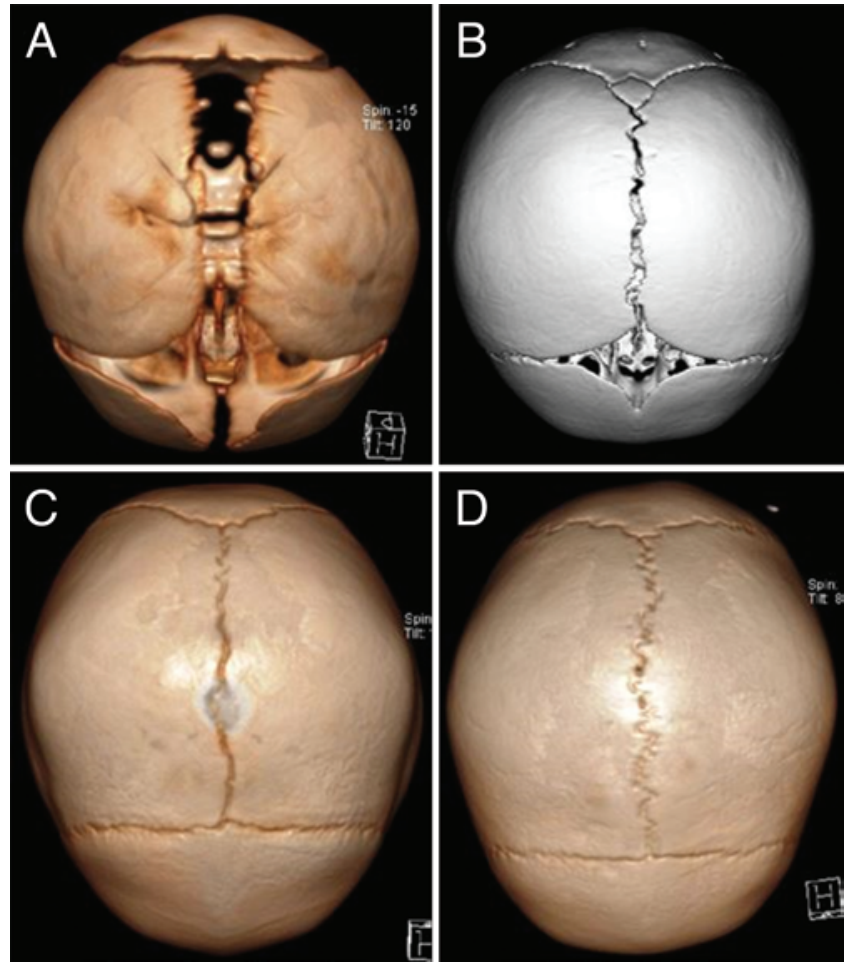

FIG. 3. 3D CT reconstructions of head CT images show normal cranial sutures in different children at birth (A), 6 months of age (B), 1 year of age (C), and 2 years of age (D). Figure is available in color online only.

reader was aware of the history of head trauma for both evaluations. Accordingly, the awareness of the history of head trauma did not bias the difference in sensitivity and specificity of the 2 evaluations. In addition, the standard of reference was established by the most experienced pediatric neuroradiologists, as is typically done in daily routine. Postmortem studies were not available in our cohort of patients.

\section{Conclusions}

Use of 2D+3D CT in combination demonstrates in-
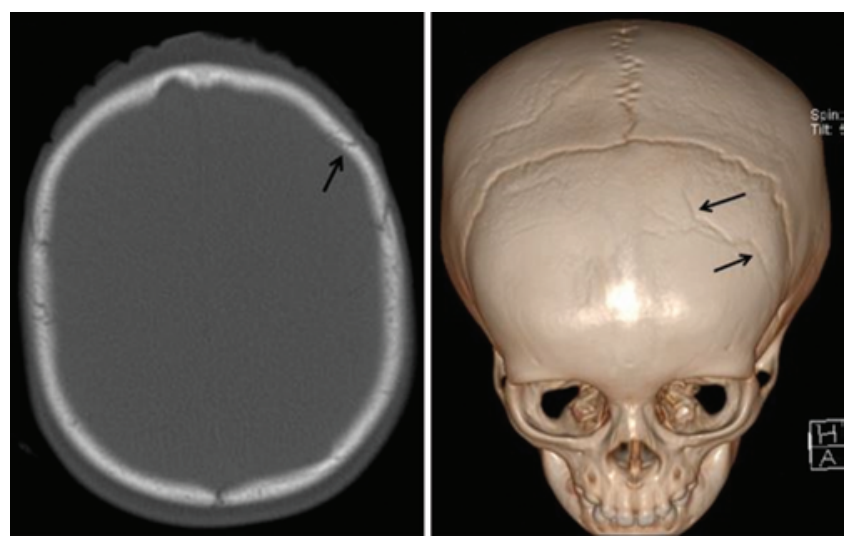

FIG. 4. Images obtained in a 20-month-old girl who presented after a motor vehicle accident. The 2D CT image (left) shows a linear lucency in the left frontal bone, possibly representing a skull fracture (arrow). The 3D CT image (right) reveals multiple vascular channels at the corresponding level (arrows). Figure is available in color online only.
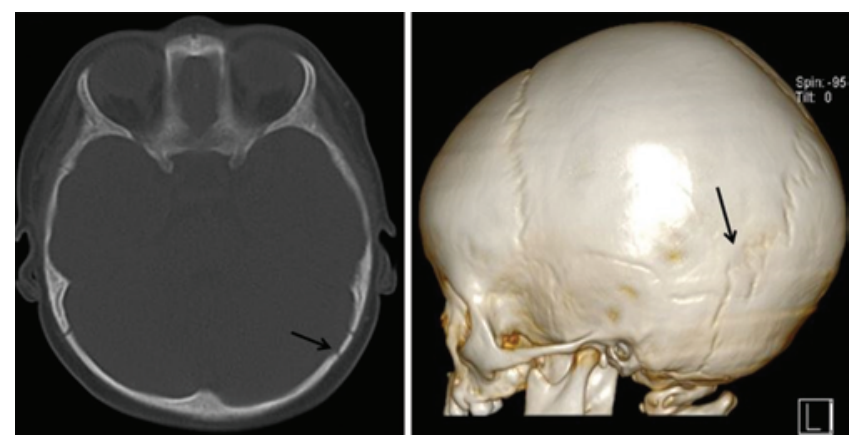

FIG. 5. CT scans in a 14-month-old girl who fell from a seat approximately $1.2 \mathrm{~m}$ in height. The 2D images (left) were suspicious for a fracture (arrow) extending to the left lambdoid suture. 3D CT (right) reveals this to represent an extension of the lambdoid suture (arrow) and ruled out a skull fracture. Figure is available in color online only.

creased sensitivity in the diagnosis of linear skull fractures in all children and increased specificity in children less than 2 years of age. In children less than 2 years of age, added confidence in the interpretation of fractures by distinguishing them from sutures may have a significant implication in the setting of nonaccidental trauma. Furthermore, 3D CT is available at no added cost, scan time, or radiation exposure, which provides trainees and clinicians with limited experience an additional valuable tool for routine imaging of pediatric head trauma.

\section{References}

1. Calhoun PS, Kuszyk BS, Heath DG, Carley JC, Fishman EK: Three-dimensional volume rendering of spiral CT data: theory and method. Radiographics 19:745-764, 1999

2. Dunning J, Daly JP, Lomas JP, Lecky F, Batchelor J, Mackway-Jones K: Derivation of the children's head injury algorithm for the prediction of important clinical events decision rule for head injury in children. Arch Dis Child 91:885-891, 2006

3. Erlichman DB, Blumfield E, Rajpathak S, Weiss A: Association between linear skull fractures and intracranial hemorrhage in children with minor head trauma. Pediatr Radiol 40:1375-1379, 2010

4. Grassberger M, Gehl A, Püschel K, Turk EE: 3D reconstruction of emergency cranial computed tomography scans as a tool in clinical forensic radiology after survived blunt head trauma-report of two cases. Forensic Sci Int 207:e19-e23, 2011

5. Greenes DS, Schutzman SA: Clinical significance of scalp abnormalities in asymptomatic head-injured infants. Pediatr Emerg Care 17:88-92, 2001

6. Keenan HT, Bratton SL: Epidemiology and outcomes of pediatric traumatic brain injury. Dev Neurosci 28:256-263, 2006

7. Kim YI, Cheong JW, Yoon SH: Clinical comparison of the predictive value of the simple skull $\mathrm{x}$-ray and 3 dimensional computed tomography for skull fractures of children. J Korean Neurosurg Soc 52:528-533, 2012

8. Mulroy MH, Loyd AM, Frush DP, Verla TG, Myers BS, Bass $\mathrm{CR}$ : Evaluation of pediatric skull fracture imaging techniques. Forensic Sci Int 214:167-172, 2012

9. Osmond MH, Klassen TP, Wells GA, Correll R, Jarvis A, Joubert G, et al: CATCH: a clinical decision rule for the use of computed tomography in children with minor head injury. CMAJ 182:341-348, 2010

10. Pinto PS, Meoded A, Poretti A, Tekes A, Huisman TA: The 
unique features of traumatic brain injury in children. review of the characteristics of the pediatric skull and brain, mechanisms of trauma, patterns of injury, complications, and their imaging findings-part 2. J Neuroimaging 22:e18-e41, 2012

11. Pinto PS, Poretti A, Meoded A, Tekes A, Huisman TA: The unique features of traumatic brain injury in children. Review of the characteristics of the pediatric skull and brain, mechanisms of trauma, patterns of injury, complications and their imaging findings - part 1. J Neuroimaging 22:e1-e17, 2012

12. Ringl H, Schernthaner R, Philipp MO, Metz-Schimmerl S, Czerny C, Weber M, et al: Three-dimensional fracture visualisation of multidetector CT of the skull base in trauma patients: comparison of three reconstruction algorithms. Eur Radiol 19:2416-2424, 2009

13. Ringl H, Schernthaner RE, Schueller G, Balassy C, Kienzl D, Botosaneanu A, et al: The skull unfolded: a cranial CT visualization algorithm for fast and easy detection of skull fractures. Radiology 255:553-562, 2010

14. Rodt T, Bartling SO, Zajaczek JE, Vafa MA, Kapapa T, Majdani O, et al: Evaluation of surface and volume rendering in 3D-CT of facial fractures. Dentomaxillofac Radiol 35:227231, 2006

15. Schutzman SA, Greenes DS: Pediatric minor head trauma. Ann Emerg Med 37:65-74, 2001

16. Simon B, Letourneau P, Vitorino E, McCall J: Pediatric minor head trauma: indications for computed tomographic scanning revisited. J Trauma 51:231-238, 2001
17. Zacharia TT, Nguyen DT: Subtle pathology detection with multidetector row coronal and sagittal CT reformations in acute head trauma. Emerg Radiol 17:97-102, 2010

\section{Author Contributions}

Conception and design: Bosemani, Poretti, Huisman. Acquisition of data: Bosemani, Orman, Seeburg, Zamora, Tekes, Huisman. Analysis and interpretation of data: Bosemani, Wagner, Zamora, Oshmyansky, Poretti, Huisman. Drafting the article: Orman. Critically revising the article: Bosemani, Wagner, Poretti, Jallo, Huisman. Reviewed submitted version of manuscript: Wagner, Seeburg, Zamora, Tekes, Poretti, Jallo, Huisman. Approved the final version of the manuscript on behalf of all authors: Bosemani. Statistical analysis: Oshmyansky. Study supervision: Bosemani, Poretti.

\section{Correspondence}

Thangamadhan Bosemani, Division of Pediatric Radiology and Section of Pediatric Neuroradiology, Russell H. Morgan Department of Radiology and Radiological Science, Charlotte R. Bloomberg Children's Center, Sheikh Zayed Tower, Rm. 4174, 1800 Orleans St., Baltimore, MD 21287-0842. email: tbosema1@ jhmi.edu. 